like graphene ${ }^{7}$, which shows potential as a transparent conductive electrode with superior flexibility for organic optoelectronics. Indeed, recent LEEM observations of $\mathrm{CuPc}$ deposited on graphene indicate $2 \mathrm{D}$ lattice gas formation ${ }^{8}$, due to the same repulsive intermolecular interaction as on $\operatorname{Ag}(111)$.

The work also opens a new field of activity for theoretical studies. In fact, the density functional theory (DFT) community has already been accounting for van der Waals interactions in organic thin film growth ${ }^{9}$; it may therefore provide a deeper insight into structural and electronic properties of heteromolecular phases ${ }^{10}$. Accessing the phase diagram itself is beyond the scope of standard DFT, but $a b$ initio atomistic thermodynamics calculations ${ }^{11}$ might provide complementary support to account for such a complicated coexistence of up to two crystalline molecular phases with a 2D lattice gas.

Christian Teichert is at the Institute of Physics of the Montanuniversitaet Leoben, Leoben 8700, Austria. e-mail:teichert@unileoben.ac.at
References

1. Hlawacek, G et al Science 321, 108-111 (2008).

2. Goiri, E. et al. Phys. Rev. Lett. 112, 117602 (2010).

3. Henneke, C., Felter, J., Schwarz, D., Tautz, F. S. \& Kumpf, C. Nat. Mater. 16, 628-633 (2017).

4. Schmidt, Th. et al. Ultramicroscopy 110, 1358-1361 (2001).

5. Huang, Y. L. et al. Small 6, 70-75 (2010)

6. Huang, H., Huang, Y., Pflaum, J., Wee, A. \& Chen, W. Appl. Phys. Lett. 95, 263309 (2009)

. Hlawacek, G., Khokhar, F. S., van Gastel, R., Poelsema, B. \& Teichert, C. Nano Lett. 11, 333-337 (2011)

8. Schwarz, D., Henneke, C. \& Kumpf, C. New. J. Phys. 18, 0234703 (2016)

9. Langreth, D. C. et al. J. Phys. Condens. Matter 21, 084203 (2009).

10. Stadtmüller, B. et al. Nat. Commun. 5, 3685 (2014)

11. Reuter, K. \& Scheffler, M. Phys. Rev. Lett. 90, 046103 (2003).

Published online: 13 March 2017

\title{
PLASTICS ON THE MENU
}

The ability of moth caterpillars to digest polyethylene, reported recently by Bombelli and colleagues ${ }^{1}$, has awakened fresh interest in using enzymes to break down plastic waste, and perhaps even to convert it to useful materials. The demand for such a process is clear. Millions of tonnes of plastics are discarded worldwide every year, much of it in packaging. Only a small proportion is recycled, and much ends up in landfill - or worse, as pollution dumped in the natural environment. The most common types of plastic in waste, such as high- and low-density polyethylene (H/LDPE), polypropylene, polyvinylchloride and polyethylene terephthalate (PET), break down only very slowly: some can take centuries, especially if buried and hidden from ultraviolet light.

The consequences are well rehearsed: not just a defacing of the environment, but a hazard to wildlife, with sea creatures ingesting or becoming entangled in plastic. Most soil and marine bacteria have no impact on such plastics.

But plastic-degrading microorganisms do exist. One such was identified by Yoshida and co-workers ${ }^{2}$ : through screening of natural microbial communities found on PET debris at a bottle recycling facility, they discovered a bacterium named Ideonella sakaiensis, which can metabolize the plastic. Microbes that break down polyethylene have also been found in the gut flora of the larval Indian mealmoth Plodia interpunctella ${ }^{3}$. And there are also fungi that can biodegrade plastics, such as the species Pestalotiopsis microspora, which infests plants in South America and Asia and is able to break down polyurethane $e^{4}$.

Given these precedents, the discovery that the greater wax moth Galleria mellonella can devour LDPE - it was serendipitously found to chew holes in plastic carrier bags - seems less surprising ${ }^{1}$. Wax moths, which are known throughout the world, are so called because of the propensity of their larvae to feed on the wax of honeybee combs: a threat against which beekeepers must take careful precautions. Wax being made from long-chain hydrocarbons (primarily aliphatic esters), it stands to reason that the larval digestive system can handle polyethylene too.

The polymer is degraded to ethylene glycol, potentially a useful chemical feedstock. And the rate of degradation - a single worm can eat through about $1 \mathrm{mg}$ in 12 hours - is rather greater than that found for previous microbial processes. It isn't yet clear if the enzymes responsible are produced by the caterpillars themselves or reside in their gut microbiome. The latter possibility might conceivably be the more promising, especially if the metabolic pathway turns out to involve more than one enzyme, since then the bacteria concerned might be cultivated as integrated plastic-degradation units rather than having to cope with a multistep process in a free-enzyme reactor. Either way, Bombelli et al.

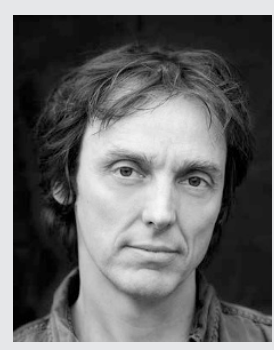

\section{PHILIP BALL}

hope that the discovery might lead to a viable biotechnological solution to plastic waste.

But a solution need not be bio-inspired. The alternative of developing purely synthetic catalysts that break down plastics, ideally to useful substances such as hydrocarbon fuels, also looks viable in principle. For example, Jia et al. have shown that two catalysts working in tandem, originally developed to link short hydrocarbons into longer ones for fuel production, can also degrade polyethylene into such long-chain fuel hydrocarbons in the presence of smaller hydrocarbon molecules 5 . The process isn't commercially viable yet, but it reminds us that nature's ingenuity shouldn't blind us to our own.

References

1. Bombelli, P., Howe, C. J. \& Bertocchini, F. Curr. Biol. 27, R283-R293 (2017).

2. Yoshida, S. et al. Science 351, 1196-1199 (2016).

3. Yang, J., Yang, Y., Wu, W.-M., Zhao, J. \& Jiang, L. Environ. Sci. Technol. 48, 13776-13784 (2014).

4. Russell, J. R. et al. Appl. Environ. Microbiol. 77, 6076-6084 (2011).

5. Jia, X., Qin, C., Friedberger, T., Guan, Z. \& Huang, Z. Sci. Adv. 2, e1501591 (2016). 